Research Article

\title{
The Influence of Orbital Element Error on Satellite Coverage Calculation
}

\author{
Guangming Dai $\left(\mathbb{D}\right.$, Xiaoyu Chen $\left(\mathbb{D}\right.$, Mingcheng Zuo $\mathbb{D}$, Lei Peng $\mathbb{D}^{\mathbb{D}}$, Maocai Wang $\mathbb{D}$, \\ and Zhiming Song $(\mathbb{D}$
}

\begin{abstract}
School of Computer Science, Hubei Key Laboratory of Intelligent Geo-Information Processing, China University of Geosciences, Lumo Road, Wuhan 430074, China
\end{abstract}

Correspondence should be addressed to Xiaoyu Chen; cxymail@126.com

Received 29 November 2017; Revised 5 April 2018; Accepted 14 May 2018; Published 5 July 2018

Academic Editor: Enrico C. Lorenzini

Copyright ( 2018 Guangming Dai et al. This is an open access article distributed under the Creative Commons Attribution License, which permits unrestricted use, distribution, and reproduction in any medium, provided the original work is properly cited.

\begin{abstract}
This paper studies the influence of orbital element error on coverage calculation of a satellite. In order to present the influence, an analysis method based on the position uncertainty of the satellite shown by an error ellipsoid is proposed. In this error ellipsoid, positions surrounding the center of the error ellipsoid mean different positioning possibilities which present three-dimensional normal distribution. The possible subastral points of the satellite are obtained by sampling enough points on the surface of the error ellipsoid and projecting them on Earth. Then, analysis cases are implemented based on these projected subastral points. Finally, a comparison report of coverage calculation between considering and not considering the error of orbital elements is given in the case results.
\end{abstract}

\section{Introduction}

The calculation of satellite and constellation coverage of ground targets is a basic problem of Earth observation systems. Various kinds of satellites and constellations are available and selectable according to different requirements of mission design. As the basic purposes of constellation design, continuous coverage and maximum regional coverage are often considered. Hence, many proposed methods were devoted to how to optimize these two goals. Cote [1] offered tips on how to maximize satellite coverage while cruising in the high-latitude West Coast waters of British Columbia. Yung and Chang [2] proposed a method for maximizing satellite coverage at predetermined local times for a set of predetermined geographic locations. Draim [3] described a new four-satellite elliptic orbit constellation giving continuous line-of-sight coverage of every point on Earth's surface.

During the implementation of a mission, the visible time windows of important targets are also usually considered. For example, Lian et al. [4] proposed two static and dynamic models of observation toward Earth by agile satellite coverage to decompose area targets into small pieces and compute visible time window subarea targets. Wang et al. [5] analyzed four parameters to determine the coverage area of satellite. It also introduced and compared two current test methods of satellite area, which presents the composition of the required test equipment and test processes, and proved the effectiveness of the tests through engineering practices. Besides the numerical analysis methods, heuristic search algorithms such as particle swarm optimization [6] were also applied to the satellite coverage calculation. Huang and Dai [7] proposed an optimal design of constellation of multiple regional coverage based on NSGA-II with the aid of multiobjective optimization.

The error influence of orbital elements on the satellite positioning was mainly considered in the field GIS and radar location [8-10]. Analysis methods proposed in these papers usually employed a model that can transform the possible position of a satellite to the points in an error ellipsoid. Nelson [11] introduced and discussed some basic properties of the error ellipsoid. For example, the basic parameters of the error ellipsoid were depicted by the axial direction and the axial ratio among each axis. In the study of satellite 
positioning error, the error ellipsoid was used in the positioning accuracy analysis of global navigation satellite system [12]. Some papers [13-15] introduced that the axis ratio of error ellipsoid for GPS should be controlled within a certain range. Wang et al. [16] selected eight sites to study the different shapes of the error ellipsoid in the global range. Alfano [17] showed the effects of positional uncertainty on the Gaussian probability computation for orbit conjunction. A model of position uncertainty for the TIS-B System was proposed [18]. Shi et al. [19] introduced a distributed location model and algorithm with position uncertainty. Trifonov [20] introduced position uncertainty measures for a particle on the sphere.

However, for coverage calculation, the influence of the orbital element error has not been considered, which is the research purpose of this paper. Because only the error influence of initial orbital elements on coverage calculation is studied in this paper, hence, the influence of other variables, such as perturbation, are not considered here. Because of the nonlinear function between satellite position and orbital elements, assuming the error of orbital elements presents normal distribution, therefore, the uncertainty of the satellite position will also present normal distribution in the threedimensional space. The relationship between the error of satellite position and orbital element error can be obtained according to the knowledge of error propagation and the function relationship between the satellite position and orbital elements, which is the basis of coverage uncertainty analysis.

The rest of this paper is organized as follows. In Section 2, the proposed method is introduced. In Section 3, preparations for cases are given. In Section 4, five analysis cases are implemented. Section 5 is devoted to the analysis of experimental results and summary of this paper. Section 6 gives some conclusions.

\section{Coverage Uncertainty Related to Orbital Elements}

The uncertainty analysis method proposed in this paper includes four steps, which are summarized as follows:

Step 1: obtain the position vector covariance matrix based on the given orbital element error matrix and transformation matrix.

Step 2: calculate the eigenvalues and eigenvectors of the position vector covariance matrix. The eigenvalue is the axial length of the error ellipsoid, and the eigenvector is the axial direction of the error ellipsoid. Then, perform the rotation and translation operations on the error ellipsoid.

Step 3: extract the edge points of the error ellipsoid projection on the surface of Earth.

Step 4: obtain the coverage analysis results to ground targets based on the extracted edge points.

In the above four steps, the implementations of steps 1 and 2 are to obtain the necessary parameters about error ellipsoid. Steps 3 and 4 are utilized to project all the points of error ellipsoid surface on Earth for the further coverage calculation. Process simulation presented in Figure 1 shows that each extracted point of the projection edge is regarded as the worst position of satellite positioning, and all the analysis work are implemented based on these points. The method employed to extract the edge points is a convex hull algorithm shown in Table 1. The analysis method metioned in step 4 is introduced in Table 2 with more details. Sections 2.1 and 2.2 describe the specific process of obtaining an error ellipsoid.

2.1. Obtain the Covariance Matrix of the Satellite Position. Considering the nonlinear relationship between the satellite position and orbital elements, the covariance matrix and propagation matrix between orbital elements and position uncertainty can only be obtained by solving partial derivative, which can be shown by

$$
\underset{m m}{D z z}=\underset{m n}{\operatorname{D}} \underset{n n}{\operatorname{Dn}} x k^{T}
$$

where $D z z_{m m}$ is an $m * m$ square matrix. In this paper, $D z z_{m m}$ represents the covariance matrix of satellite position in directions $X, Y$, and $Z . D x x_{n n}$ is the error matrix of orbital elements.

When $z$ is a nonlinear function of $x$, such as

$$
\begin{gathered}
z_{1}=f_{1}\left(x_{1}, x_{2}, \ldots, x_{n}\right), \\
z_{2}=f_{2}\left(x_{1}, x_{2}, \ldots, x_{n}\right), \\
\vdots \\
z_{m}=f_{m}\left(x_{1}, x_{2}, \ldots, x_{n}\right),
\end{gathered}
$$

the partial derivative of $z$ by partial derivative of $x$ needs to be obtained by the following method:

$$
\begin{gathered}
d z_{1}=\frac{\partial f_{1}}{\partial x_{1}} d x_{1}+\frac{\partial f_{1}}{\partial x_{2}} d x_{2}+\cdots+\frac{\partial f_{1}}{\partial x_{n}} d x_{n}, \\
d z_{2}=\frac{\partial f_{2}}{\partial x_{1}} d x_{1}+\frac{\partial f_{2}}{\partial x_{2}} d x_{2}+\cdots+\frac{\partial f_{2}}{\partial x_{n}} d x_{n} \\
\vdots \\
d z_{m}=\frac{\partial f_{t}}{\partial x_{1}} d x_{1}+\frac{\partial f_{t}}{\partial x_{2}} d x_{2}+\cdots+\frac{\partial f_{t}}{\partial x_{n}} d x_{n} .
\end{gathered}
$$

Then, (5) can be obtained based on (3) and represented by

$$
\begin{gathered}
\underset{m 1}{z}=\left(\begin{array}{c}
z_{1} \\
z_{2} \\
\vdots \\
z_{m}
\end{array}\right), \\
\underset{m 1}{z}=\left(\begin{array}{c}
d z_{1} \\
d z_{2} \\
\vdots \\
d z_{m}
\end{array}\right),
\end{gathered}
$$




$$
k=\left(\begin{array}{ccc}
\frac{\partial f_{1}}{\partial x_{1}} \frac{\partial f_{1}}{\partial x_{2}} & \cdots & \frac{\partial f_{1}}{\partial x_{n}} \\
\frac{\partial f_{2}}{\partial x_{1}} \frac{\partial f_{2}}{\partial x_{2}} & \cdots & \frac{\partial f_{2}}{\partial x_{n}} \\
\vdots & & \vdots \\
\frac{\partial f_{t}}{\partial x_{1}} \frac{\partial f_{t}}{\partial x_{2}} & \cdots & \frac{\partial f_{m}}{\partial x_{n}}
\end{array}\right) .
$$

The nonlinear function between the satellite position vector and orbital elements is shown in (6), (7), and (8), where $a$, $e, E, i, \Omega$, and $\omega$ are orbital elements and $P$ and $Q$ are functions of $\Omega, \omega$, and $i$. Vector $r$ is decomposed into three vectors in the directions $x, y$, and $z$, and in each direction, the partial derivatives of orbital elements can be obtained. For example, the partial derivative of $z$ by that of $a, e, E, i, \Omega$, and $\omega$ can be presented in (9). Similarly, gaining the partial derivatives of $x$ and $y$ by that of $a, e, E, i, \Omega$, and $\omega$, respectively, can finally obtain the error propagation matrix.

$$
\begin{gathered}
r=a(\cos E-e) \cdot P+a \sqrt{1-e^{2}} \sin E \cdot Q, \\
P=\left(\begin{array}{c}
\cos \Omega \cos \omega-\sin \Omega \sin \omega \cos i \\
\sin \Omega \cos \omega+\cos \Omega \sin \omega \cos i \\
\sin \omega \sin i
\end{array}\right), \\
Q=\left(\begin{array}{c}
-\cos \Omega \sin \omega-\sin \Omega \cos \omega \cos i \\
-\sin \Omega \sin \omega+\cos \Omega \cos \omega \cos i \\
\cos \omega \sin i
\end{array}\right),
\end{gathered}
$$

$\frac{\partial_{z}}{\partial_{a}}=\sin E \cos \Omega \sqrt{1-e^{2}}-\sin \Omega \sin i(e-\cos E)$,

$\frac{\partial_{z}}{\partial_{e}}=a \sin \Omega \sin i-\frac{a e \sin E \cos \Omega \sin i}{\sqrt{1-e^{2}}}$,

$\frac{\partial_{z}}{\partial_{E}}=a \cos E \cos \Omega \sin i \sqrt{1-e^{2}}-a \sin E \sin \Omega \sin i$,

$\frac{\partial_{z}}{\partial_{i}}=a \sin E \cos \Omega \sqrt{1-e^{2}}-a \cos i \sin \Omega(e-\cos E)$,

$\frac{\partial_{z}}{\partial_{\Omega}}=-a \cos \Omega \sin i(e-\cos E)-a \sin E \sin \Omega \sin i \sqrt{1-e^{2}}$,

$\frac{\partial_{z}}{\partial_{\omega}}=0$.

2.2. Rotation and Translation of the Error Ellipsoid. Assume that the major axis direction of error ellipsoid is

$$
r=\left(x_{m}, y_{m}, z_{m}\right) \text {. }
$$

So the rotation angle of major axis around axis $z$ should be

$$
\theta_{z}=\arctan \left(\frac{y_{m}}{x_{m}}\right)
$$

Then, the rotation angle of major axis around axis $y$ is

$$
\theta_{y}=\arcsin \left(\frac{\left|z_{m}\right|}{r_{m}}\right)
$$

Subsequently, all points need to be rotated around axis $x$. Based on the previous rotations, the third rotation angle is equal to the angle between axis $y$ and middle axis of error ellipsoid.

Finally, if the standard position of satellite is $(x, y, z)$, the point $\left(x_{0}, y_{0}, z_{0}\right)$ needs to be translated to a new position $\left(x_{0}+x, y_{0}+y, z_{0}+z\right)$.

2.3. Feature of the Error Ellipsoid. It is assumed that the measurement errors of six orbital elements present normal distribution. So the position error of satellite also presents threedimensional normal distribution $[5,16]$. The distribution density of possible satellite position in three-dimensional space is

$$
\frac{\exp \left(-0.5\left(r^{T} D_{z z}{ }^{-1} r\right)\right)}{(2 \pi)^{1.5} \sqrt{\Delta}}
$$

where $r$ is the position increment.

The denominator of (13) is a constant, while the numerator is changeable. So all points with the same distribution density can be presented by

$$
r^{T} D_{z z}{ }^{-1} r=k^{2} .
$$

Equation (14) is a presentation of ellipsoid cluster, and $k$ is a magnification factor. If $r$ is symbolized by

$$
r=\left(\begin{array}{c}
\Delta x \\
\Delta y \\
\Delta z
\end{array}\right),
$$

(14) can also be shown by

$$
\left[\begin{array}{c}
\Delta x \\
\Delta y \\
\Delta z
\end{array}\right]^{T}\left[\begin{array}{ccc}
\sigma_{x x}^{2} & \sigma_{x y} & \sigma_{x z} \\
\sigma_{x y} & \sigma_{y y}^{2} & \sigma_{y z} \\
\sigma_{x z} & \sigma_{y z} & \sigma_{z z}^{2}
\end{array}\right]\left[\begin{array}{c}
\Delta x \\
\Delta y \\
\Delta z
\end{array}\right]=k^{2} .
$$

Because it is not convenient to show the ellipsoid in coordinate system $O X Y Z$, the principal axis coordinate system $O U V W$ is employed to present it. For a real symmetric matrix $D_{z z}$, an orthogonal matrix $T$ can be utilized to make

$$
\left[\begin{array}{c}
\Delta x \\
\Delta y \\
\Delta z
\end{array}\right]=T\left[\begin{array}{c}
U \\
V \\
W
\end{array}\right],
$$




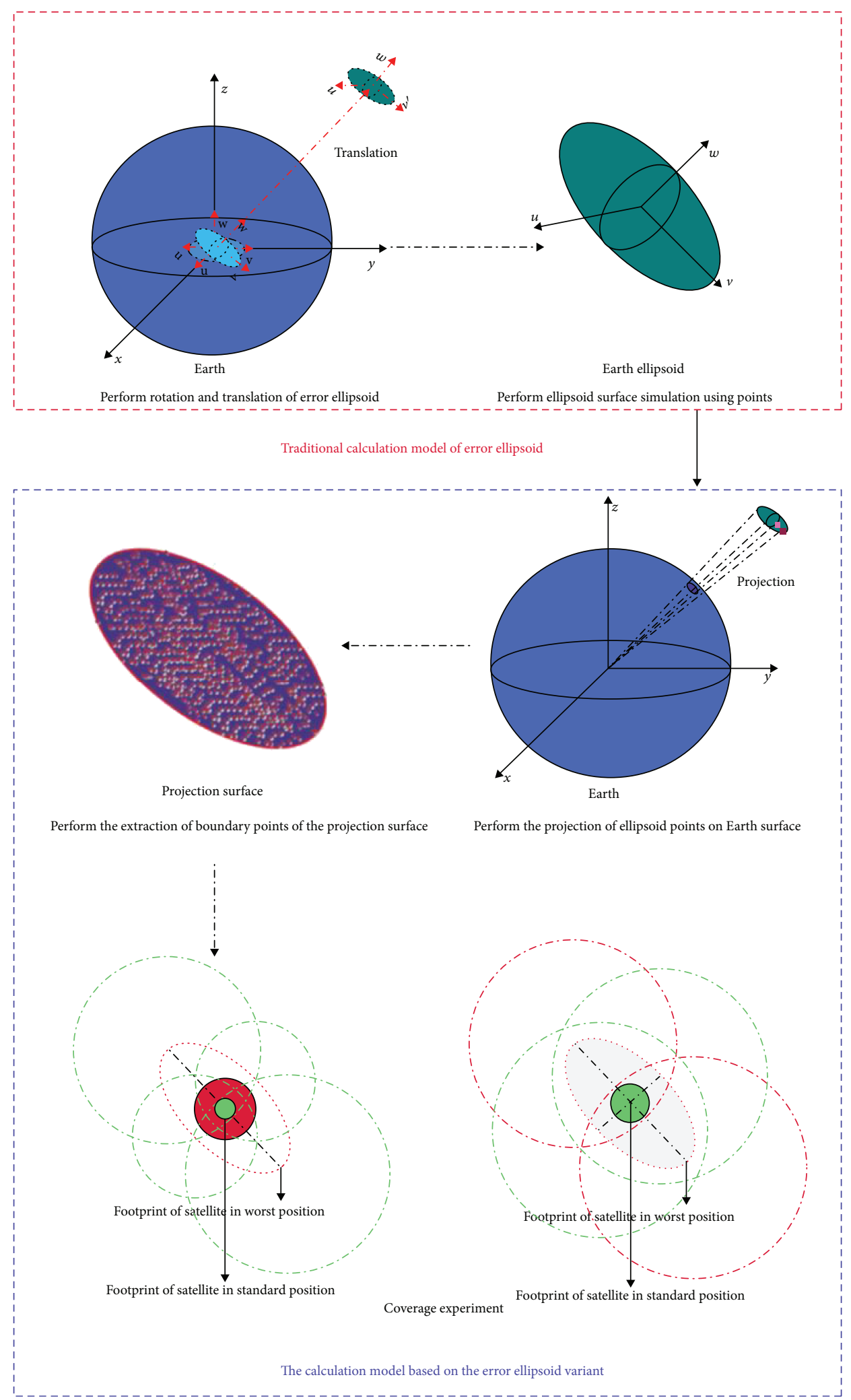

FIGURE 1: Simulation of uncertainty analysis process. 
TABLE 1: Convex hull algorithm.

Input: Planar points set $p$; Output: convex hull \#(p) of points set $p$; clockwise queue of $\#(p)$.

Step 1: Find point $p_{0}$ with the minimum ordinate in $p$. If more than one point meets this requirement, select the point with minimum horizontal ordinate.

Step 2: Order all the points according to the polar angle relative to $p_{0}$. If more than one point locates on the same direction, retain the farthest one.

Step 3: Start from the minimum polar angle, and check whether the points in this direction can be contained in \#(p). If not, put this point to \#(upper) and update it.

Step 4: Repeat step 3 to all the points have been checked.

TABLE 2: Method of analyzing error influence.

Step 1: Find two points $p_{l 1}$ and $p_{l 2}$ from $\#(p)$ with the longest distance. Find two other points $p_{s 1}$ and $p_{s 2}$ which are perpendicular to the line $p_{l 1} p_{l 2}$.

Step 2: If The target is a point.

Step 3: Obtain the calculation result based on the points gained in step 1.

Step 4: If The target cannot be covered when subastral points are $p_{s 1}$ and $p_{s 2}$.

Step 5: Draw the conclusion: This target cannot be covered completely.

Step 6: Else If The target cannot be covered when subastral points are $p_{s 1}$ and $p_{s 2}$.

Step 7: $\quad$ Draw the conclusion: This target may be covered possibly.

Step 8: $\quad$ Else Draw the conclusion: This target can be covered completely.

Step 9: ELSE Obtain the calculation result based on the points gained in step 1.

Step 10: If The target cannot be covered when subastral points are $p_{s 1}$ and $p_{s 2}$.

Step 11: Draw the conclusion: This target cannot be covered completely.

Step 12: $\quad$ Else If The target cannot be covered when subastral points are $p_{l 1}$ and $p_{l 2}$.

Step 13: Obtain the least percentage that the area target can be covered.

Step 14: $\quad$ Else Draw the conclusion: This target can be covered completely.

and

$$
T^{T} D z z T=\left[\begin{array}{lll}
\lambda_{1} & & \\
& \lambda_{2} & \\
& & \lambda_{3}
\end{array}\right]
$$

According to (18), (15) can be shown by

$$
\left[\begin{array}{c}
U \\
V \\
W
\end{array}\right]^{T}\left[\begin{array}{ccc}
\frac{1}{\lambda_{1}} & & \\
& \frac{1}{\lambda_{2}} & \\
& & \frac{1}{\lambda_{3}}
\end{array}\right]\left[\begin{array}{c}
U \\
V \\
W
\end{array}\right]=k^{2} .
$$

Equation (19) can be expanded to

$$
\frac{u^{2}}{\lambda_{1}}+\frac{V^{2}}{\lambda_{2}}+\frac{W^{2}}{\lambda_{3}}=k^{2}
$$

Assume that each axis of the error ellipsoid is symbolized by $a, b$, and $c$; hence,

$$
\begin{aligned}
& a^{2}=k^{2} \lambda_{1}, \\
& b^{2}=k^{2} \lambda_{2}, \\
& c^{2}=k^{2} \lambda_{3} .
\end{aligned}
$$

As mentioned before, the position of the satellite is an uncertainty position distribution within error ellipsoid interior. This distribution probability can be presented by

$$
p=\iiint \frac{\exp \left(-0.5\left(U^{2} / \sigma_{1}^{2}+V^{2} / \sigma_{2}^{2}+W^{2} / \sigma_{3}^{2}\right)\right)}{(2 \pi)^{1.5} \sigma_{1} \sigma_{2} \sigma_{3}} d U d V d W
$$

giving a variable substitution, such as

$$
\begin{aligned}
& u^{\prime}=\frac{u}{\sqrt{2} \sigma 1}, \\
& v^{\prime}=\frac{v}{\sqrt{2} \sigma 2}, \\
& w^{\prime}=\frac{w}{\sqrt{2} \sigma 2} .
\end{aligned}
$$

The probability in the ellipsoid is converted to the probability in the ball as follows:

$p=\frac{1}{\pi^{1.5}} \int_{0}^{2 \pi} d \theta \int_{0}^{\pi} d \varphi \int_{0}^{k / \sqrt{2}} \exp \left(-r^{2} r^{2}\right) \sin \varphi d r=\frac{4}{\sqrt{\pi}} \int_{0}^{k / \sqrt{2}} e^{-r^{2}} r^{2} d r$.

In addition, considering that

$$
\begin{aligned}
e^{-r^{2}} & =1-r^{2}+\frac{r^{4}}{2 !}-\frac{r^{6}}{3 !}+\cdots+(-1)^{-1} \frac{r^{2 n}}{n !}+\cdots, \\
r & \in(-\infty,+\infty),
\end{aligned}
$$


TABLe 3: Data of orbital elements.

\begin{tabular}{lccccc}
\hline $\begin{array}{l}\text { Semimajor axis } \\
(\mathrm{km})\end{array}$ & Eccentricity & $\begin{array}{c}\text { True anomaly } \\
\left({ }^{\circ}\right)\end{array}$ & $\begin{array}{c}\text { Inclination of orbit } \\
\left({ }^{\circ}\right)\end{array}$ & $\begin{array}{c}\text { Argument of perigee } \\
\left({ }^{\circ}\right)\end{array}$ & $\begin{array}{c}\text { Right ascension of ascending node } \\
\left({ }^{\circ}\right)\end{array}$ \\
\hline 10,424 & 0.3 & 0 & 45 & 45 & 20 \\
\hline
\end{tabular}

TABle 4: Data of axis length.

\begin{tabular}{lcc}
\hline Major axis $(\mathrm{km})$ & Middle axis $(\mathrm{km})$ & Minor axis $(\mathrm{km})$ \\
\hline 20.9535 & 14.6836 & 5.4130 \\
\hline
\end{tabular}

(24) can be expanded to

$$
p=\frac{4}{\sqrt{2} \pi}\left(\frac{k^{3}}{6}-\frac{k^{5}}{20}+\frac{k^{7}}{112}-\frac{k^{9}}{864}+\cdots\right)
$$

where the probability of the point distribution in the ellipsoid varies with the change of $K$ as follows:

(i) When $K=1$, the probability of the point distribution in the error ellipsoid is $19.9 \%$.
TABLE 5: Data of rotation angle.

Rotation angle of the Rotation angle of the Rotation angle of the major axis around major axis around middle axis around

\begin{tabular}{lcc}
$z$-axis $\left({ }^{\circ}\right)$ & $y$-axis $\left({ }^{\circ}\right)$ & $x$-axis $\left({ }^{\circ}\right)$ \\
\hline 55.262168 & -29.999022 & 125.621076 \\
\hline
\end{tabular}

(ii) When $K=2$, the probability of the point distribution in the error ellipsoid is $73.9 \%$.

(iii) When $K=3$, the probability of the point distribution in the error ellipsoid is $95 \%$.

\section{Preparation for Coverage Case}

3.1. Calculation of the Error Ellipsoid. According to the measurement accuracy in actual engineering project, the error matrix of orbital elements is set as follows:

$$
D x x=\left(\begin{array}{cccccc}
3^{2} & 0 & 0 & 0 & 0 & 0 \\
0 & 0.002^{2} & 0 & 0 & 0 & 0 \\
0 & 0 & \left(0.05^{*} H\right)^{2} & 0 & 0 & 0 \\
0 & 0 & 0 & \left(0.05^{*} H\right)^{2} & 0 & 0 \\
0 & 0 & 0 & 0 & \left(0.05^{*} H\right)^{2} & 0 \\
0 & 0 & 0 & 0 & 0 & \left(0.05^{*} H\right)^{2}
\end{array}\right) \text {, }
$$

where $H$ is a conversion factor between angle and radian.

The orbital elements of a satellite are shown in Table 3. In addition, as a requirement of parameter transformation, the conversion relationship between eccentric anomaly $E$ and true anomaly $f$ is needed:

$$
E=2 \cdot a \tan \left(\frac{\tan (f / 2)}{((1+e) /(1-e))^{0.5}}\right) .
$$

The covariance matrix of the satellite position $D z z$ is obtained as follows:

$$
D z z=\left(\begin{array}{ccc}
264.2010 & 99.6929 & 30.6575 \\
99.6929 & 251.3009 & 168.8499 \\
30.6575 & 168.8499 & 168.4551
\end{array}\right) \text {. }
$$

The feature vector $v$ of $D z z$ is

$$
v=\left(\begin{array}{ccc}
0.4935 & 0.8516 & 0.1766 \\
0.7117 & -0.2786 & -0.6449 \\
0.5000 & -0.4439 & 0.7436
\end{array}\right)
$$

The data of axis length and rotation angle are shown in Tables 4 and 5, respectively. The standard position coordinate of the satellite position is $(3600.6,5193.1,3648.4)$. The final simulation of the error ellipsoid with different views is shown in Figure 2.

3.2. Edge Extraction of Projection on Earth. The projection of error ellipsoid on Earth is a curved surface, so in order to obtain the edge points, a mapping on plane is more convenient. 421 edge points are obtained in the case by using convex hull algorithm, with different views of simulation results shown in Figure 3. 
Three- dimensional view

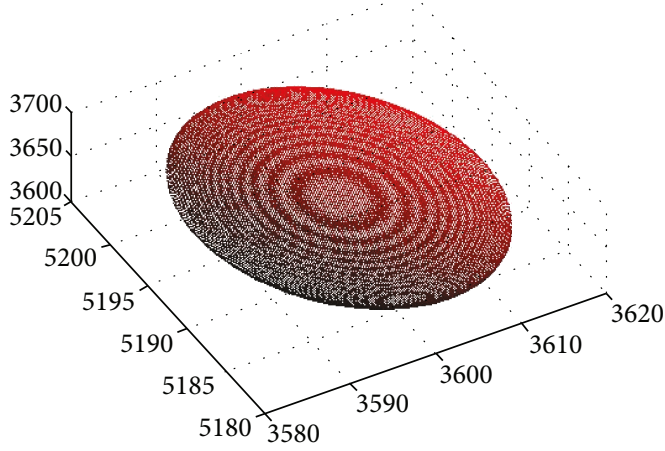

Three- dimensional view

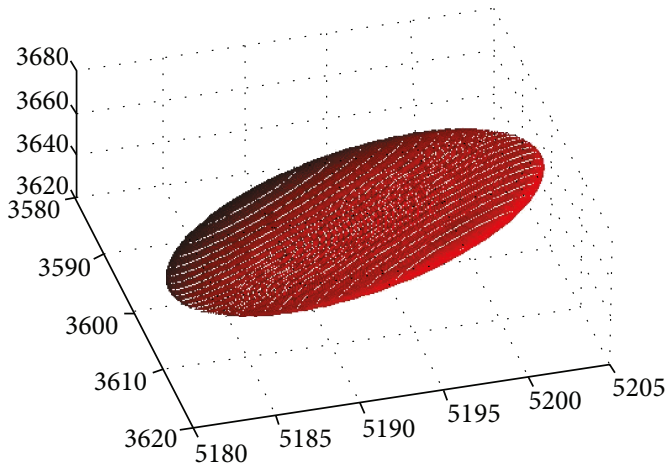

Three- dimensional view

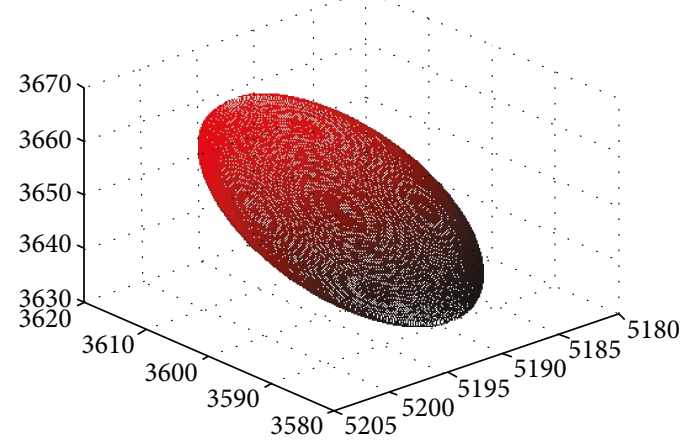

Three- dimensional view

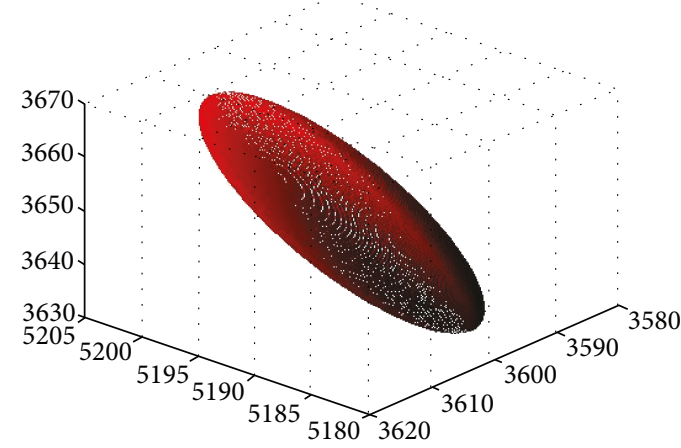

FIGURE 2: Space distribution of error ellipsoid.
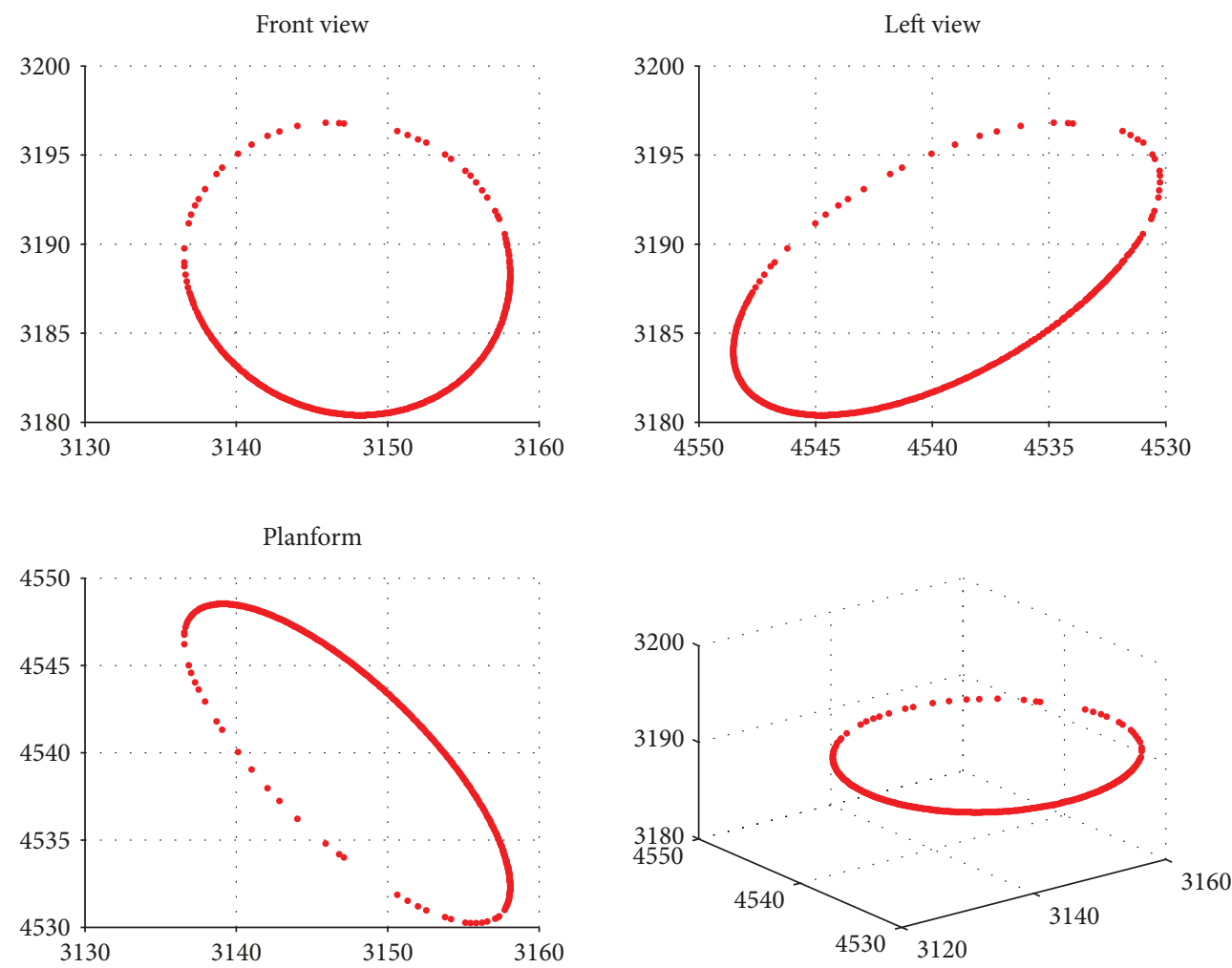

Figure 3: Result of edge extraction. 

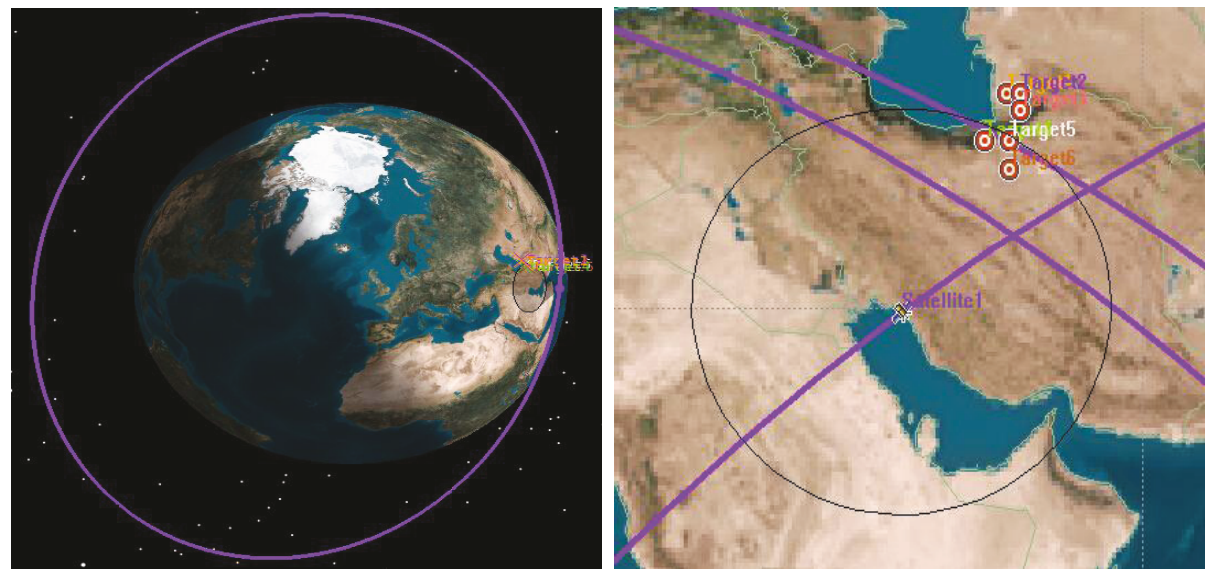

FIGURE 4: Satellite orbit and point targets.

TABLE 6: Coordinate of the six point targets.

\begin{tabular}{lcc}
\hline Group & Coordinate & Latitude and longitude \\
\hline 1 & $(3700.600,5193.100,3648.400)$ & $54.526^{\circ} \mathrm{E}, 37.855^{\circ} \mathrm{N}$ \\
2 & $(3700.600,5293.100,3648.400)$ & $55.041^{\circ} \mathrm{E}, 37.848^{\circ} \mathrm{N}$ \\
3 & $(3700.600,5293.100,3748.400)$ & $55.041^{\circ} \mathrm{E}, 37.260^{\circ} \mathrm{N}$ \\
4 & $(3900.600,5293.100,3748.400)$ & $53.612^{\circ} \mathrm{E}, 36.112^{\circ} \mathrm{N}$ \\
5 & $(3900.600,5493.100,3748.400)$ & $54.621^{\circ} \mathrm{E}, 36.117^{\circ} \mathrm{N}$ \\
6 & $(3900.600,5493.100,3948.400)$ & $54.621^{\circ} \mathrm{E}, 35.068^{\circ} \mathrm{N}$ \\
\hline
\end{tabular}

TABLE 7: Results of case 1.

\begin{tabular}{lcc}
\hline Group & $\begin{array}{c}\text { Geocentric angle without } \\
\text { considering error }\left(^{\circ}\right)\end{array}$ & $\begin{array}{c}\text { Geocentric angle with } \\
\text { considering error }\left(^{\circ}\right)\end{array}$ \\
\hline 1 & 1.356686 & 1.580021 \\
2 & 1.595898 & 1.713935 \\
3 & 1.940826 & 2.012707 \\
4 & 4.564908 & 4.720740 \\
5 & 6.070766 & 6.134848 \\
6 & 7.341740 & 7.391859 \\
\hline
\end{tabular}

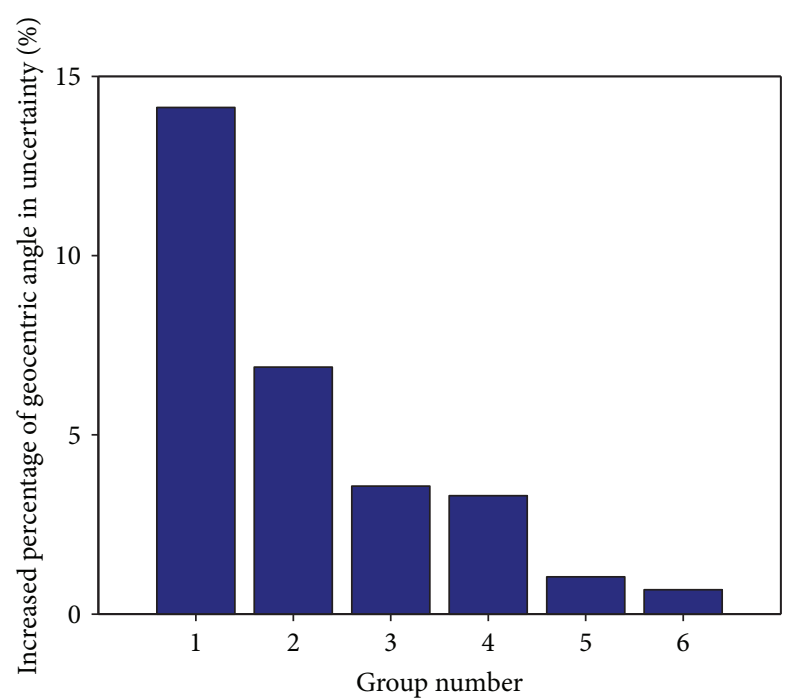

FIGURE 5: Simulation comparison of case results.

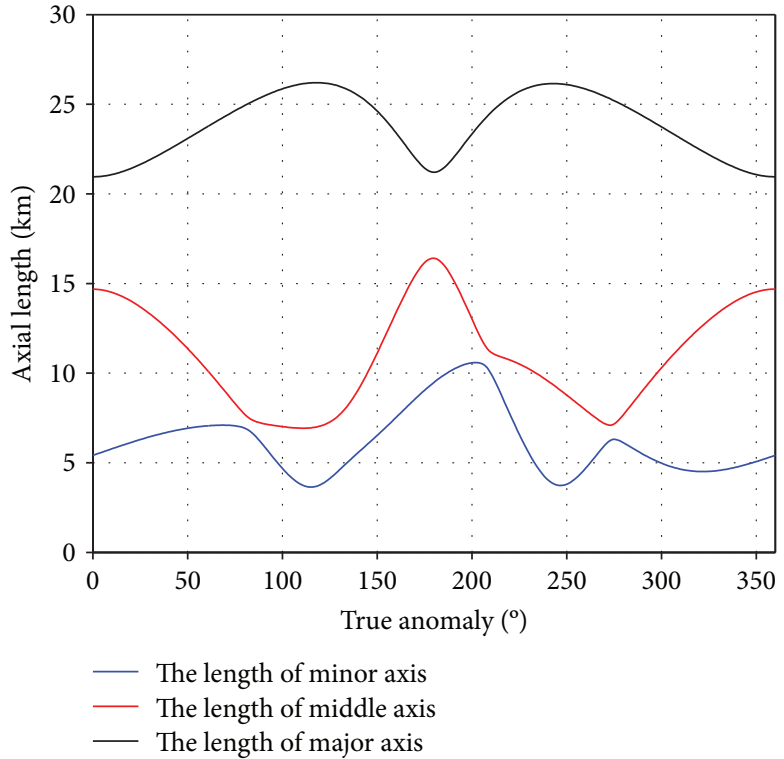

Figure 6: Changes of axial length.

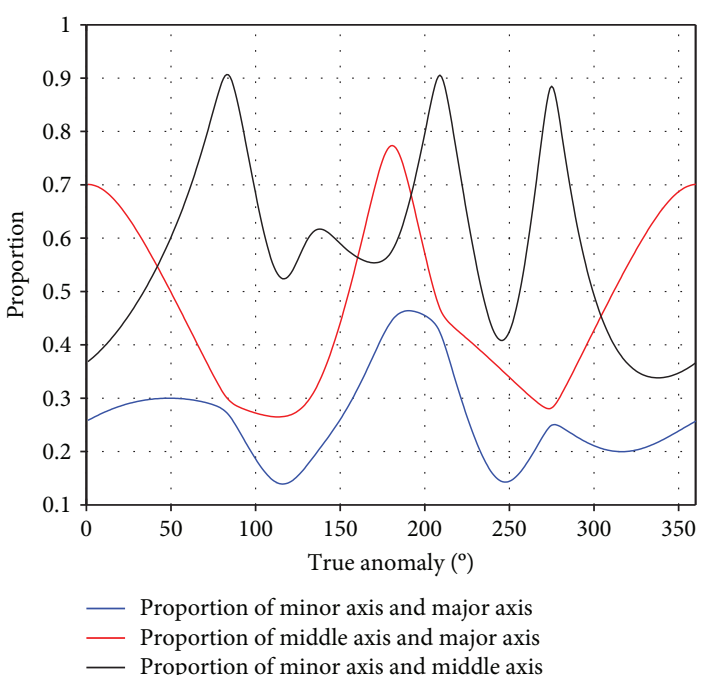

Figure 7: Changes of axial ratio. 
TABLE 8: Orbital element of each satellite.

\begin{tabular}{lccccc}
\hline $\begin{array}{l}\text { Semi major axis } \\
(\mathrm{km})\end{array}$ & Eccentricity & $\begin{array}{c}\text { True anomaly } \\
\left({ }^{\circ}\right)\end{array}$ & $\begin{array}{c}\text { Inclination of orbit } \\
\left({ }^{\circ}\right)\end{array}$ & $\begin{array}{c}\text { Argument of perigee } \\
\left({ }^{\circ}\right)\end{array}$ & $\begin{array}{c}\text { Right ascension of ascending node } \\
\left({ }^{\circ}\right)\end{array}$ \\
\hline 10,424 & 0.3 & 0 & 45 & 45 & 20 \\
10,424 & 0.3 & 0 & 45 & 45 & 40 \\
10,424 & 0.3 & 0 & 45 & 45 & 60 \\
\hline
\end{tabular}

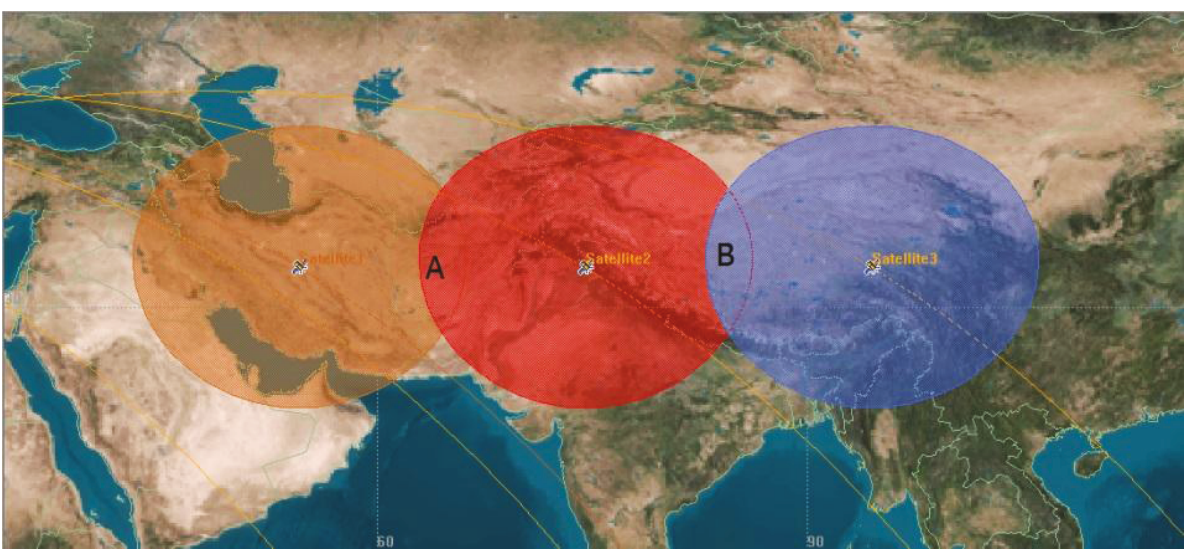

Figure 8: Positions of points A and B.

\section{Numerical Case}

Five cases with different configures are designed and implemented in this paper. Firstly, the uncertainty influence of single satellite positing on multiple point target coverage is given in case 1. Then, in order to deeply understand the shape change of "error ellipsoid" when the satellite moves to different positions, the changes of axial length and axial ratio are given in case 2 . Lastly, the uncertainty influence of a constellation positioning on multiple point targets, constellation positioning on a single area target, and constellation positioning on two area targets are given in cases 3 to 5 , respectively.

The reference system used in this paper is ECI. When the satellite is in the initial position perigee, the latitude and longitude of the satellite are $55.2^{\circ} \mathrm{E}$ and $30^{\circ} \mathrm{N}$, respectively. The orbit and ground point targets are shown in Figure 4 with $3 \mathrm{D}$ and $2 \mathrm{D}$ views.

4.1. Influence of Single Satellite Position Uncertainty on Multiple Point Target Coverage. The purpose of case 1 is to explain the necessity of considering orbital element error in coverage calculation. Six point targets are set in this case, whose coordinates are presented in Table 6, obtaining the different minimum coverage requirements of these point targets in terms of geocentric angles considering and not considering positioning uncertainty, respectively. Case results listed in Table 7 show that the geocentric angle needs to be larger to cover all the aimed targets when considering error of orbital elements. The results shown in Figure 5 obviously present the relative percentage of needed geocentric angle when considering uncertainty.
TABLE 9: Minimum coverage radius of satellites.

\begin{tabular}{lcc}
\hline Satellite & Point target & Minimum covering radius $(\mathrm{km})$ \\
\hline 1 & A & 469.590510 \\
2 & A & 1484.017928 \\
2 & B & 460.448878 \\
3 & B & 1459.206737 \\
\hline
\end{tabular}

4.2. Shape Change of the "Error Ellipsoid." The purpose of case 2 is to research on the shape change of the error ellipsoid. Analyze the axial length variation while true anomaly changes from $0^{\circ}$ to $360^{\circ}$. Focus on the shape change by studying the variations of axial length and axial ratio, which are shown in Figures 6 and 7, respectively. Interesting conclusions can be drawn from the case results. The most significant one is that the 3D shape of error ellipsoid is a timedependent function, and the error ellipsoid is rather slender when true anomaly changes around $110^{\circ}$. While anomaly reaches around $180^{\circ}$, the shape is stouter.

4.3. Uncertainty Influence of a Constellation Positioning on Multiple Point Targets. The implementation of case 3 is to study the coverage uncertainty of constellation containing 3 satellites whose orbital elements are shown in Table 8. Two point targets A $(3159.6162,5472.44908$, and 3648.4) and B (1097.44509, 6222.5133, and 3648.4) shown in Figure 8 are set in this case. The latitude and longitude of these two points are $\left(60^{\circ} \mathrm{E}, 30^{\circ} \mathrm{N}\right)$ and $\left(80^{\circ} \mathrm{E}, 30^{\circ} \mathrm{N}\right)$, respectively. Case results in Table 9 show that when considering positioning uncertainty, the minimum required covering radius is larger than that of not considering uncertainty. If it is needed to cover both 
TABLE 10: Coverage results of a circular area when the sensor angle is $7^{\circ}$.

\begin{tabular}{lccccccc}
\hline Coverage times & Target number & Coverage times & Target number & Coverage times & Target number & Coverage times & Target number \\
\hline 31 & 2525 & 37 & 404 & 43 & 0 & 49 & 0 \\
32 & 2780 & 38 & 193 & 44 & 0 & 50 & 0 \\
33 & 1918 & 39 & 38 & 45 & 0 & 51 & 0 \\
34 & 1398 & 40 & 0 & 0 & 46 & 0 & 52 \\
35 & 1082 & 41 & 0 & 48 & 0 & 53 & 0 \\
36 & 818 & 42 & &
\end{tabular}

TABLE 11: Coverage results of a circular area when the sensor angle is $7.4^{\circ}$.

\begin{tabular}{lccccccc}
\hline Coverage times & Target number & Coverage times & Target number & Coverage times & Target number & Coverage times & Target number \\
\hline 31 & 1810 & 37 & 2145 & 43 & 1392 & 49 & 821 \\
32 & 5354 & 38 & 4878 & 44 & 2160 & 50 & 724 \\
33 & 5371 & 39 & 3507 & 45 & 1268 & 51 & 516 \\
34 & 3882 & 40 & 2590 & 46 & 1316 & 52 & 344 \\
35 & 3745 & 41 & 2006 & 47 & 1331 & 53 & 215 \\
36 & 4106 & 42 & 2102 & 48 & 1061 & 54 & 38,589 \\
\hline
\end{tabular}

TABLE 12: Coverage results of a circular area when the sensor angle is $7.7^{\circ}$.

\begin{tabular}{lccccccc}
\hline Coverage times & Target number & Coverage times & Target number & Coverage times & Target number & Coverage times & Target number \\
\hline 31 & 0 & 37 & 902 & 43 & 675 & 49 & 501 \\
32 & 0 & 38 & 836 & 44 & 1185 & 50 & 469 \\
33 & 0 & 39 & 1756 & 45 & 612 & 51 & 257 \\
34 & 0 & 40 & 1441 & 46 & 1160 & 52 & 53 \\
35 & 0 & 41 & 1179 & 47 & 775 & 59 \\
36 & 198 & 42 & 1152 & 48 & 440 & 54 & 52,792 \\
\hline
\end{tabular}

TABLE 13: Coverage results of a circular area when the sensor angle is $8^{\circ}$.

\begin{tabular}{|c|c|c|c|c|c|c|c|}
\hline Coverage times & Target number & Coverage times & Target number & Coverage times & Target number & Coverage times & Target number \\
\hline 31 & 0 & 37 & 0 & 43 & 0 & 49 & 0 \\
\hline 32 & 0 & 38 & 0 & 44 & 0 & 50 & 0 \\
\hline 33 & 0 & 39 & 0 & 45 & 0 & 51 & 0 \\
\hline 34 & 0 & 40 & 0 & 46 & 0 & 52 & 0 \\
\hline 35 & 0 & 41 & 0 & 47 & 0 & 53 & 0 \\
\hline 36 & 0 & 42 & 0 & 48 & 0 & 54 & 96,597 \\
\hline
\end{tabular}

points $\mathrm{A}$ and $\mathrm{B}$ at the same time, the minimum radius must be $1484.017928 \mathrm{~km}$.

4.4. Uncertainty Influence of Constellation Positioning on a Single Area Target. We have mentioned that 421 edge points are obtained in the case by using convex hull algorithm. In order to reduce the calculation expense, 54 edge points are chosen evenly to implement the coverage case containing 96,576 ground target points. Only when each of the 96,576 sampling points can be covered by the satellite, whose subastral point is any one of the 54 edge points, the area is deemed covered completely. Assuming that the target is a circular area, and the radius of this area is $100 \mathrm{~km}$, compute the minimum sensor angle meeting the coverage requirements of this area when the angle of the satellite sensor changes from $7^{\circ}$. Case results are shown in Tables 10,11, 12, and 13. In order to present this change significantly, relevant simulation results of these data are shown in Figures 9-12, respectively. As well, the percentage change of area coverage is also shown in Figure 13.

4.5. Uncertainty Influence of Constellation Positioning on Two Area Targets. Assuming that A and B are circular areas with circle radius $=100 \mathrm{~km}$. The minimum coverage radius 


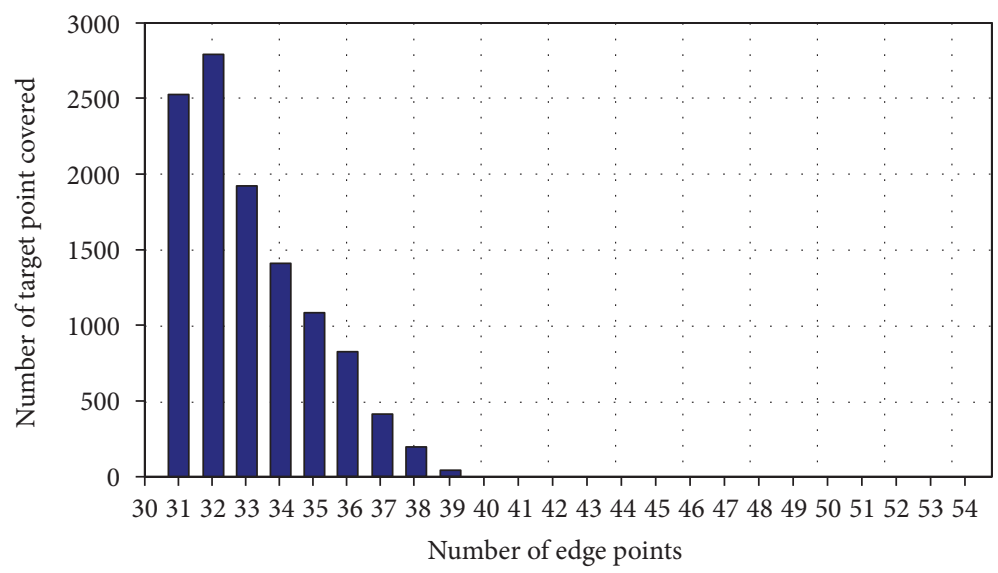

Figure 9: Coverage results of a circular area when the sensor angle is $7^{\circ}$.

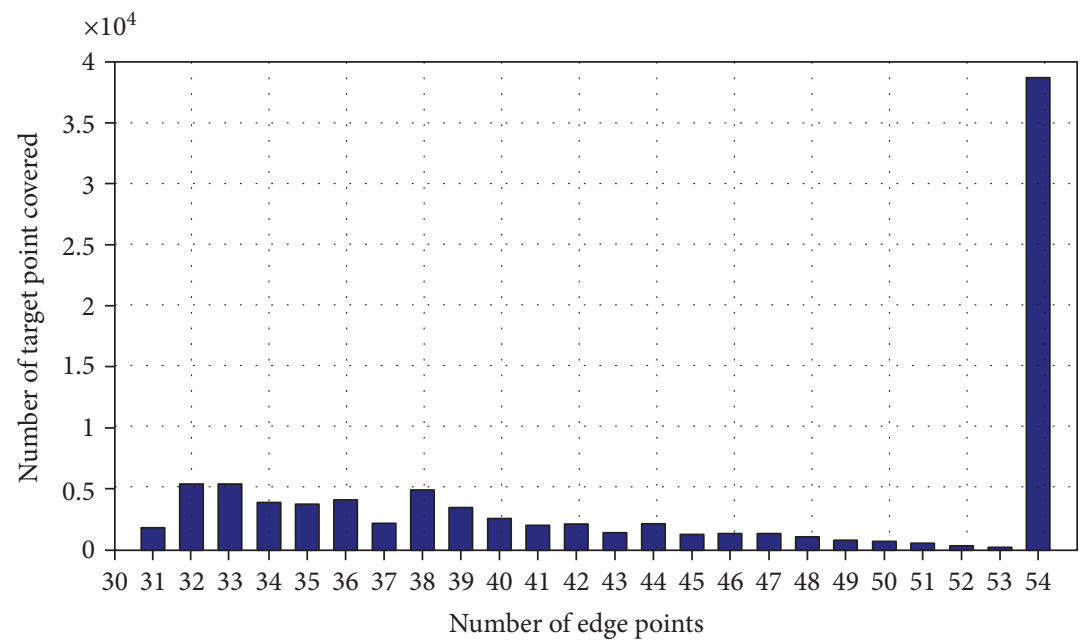

FIGURE 10: Coverage results of a circular area when the sensor angle is $7.4^{\circ}$.

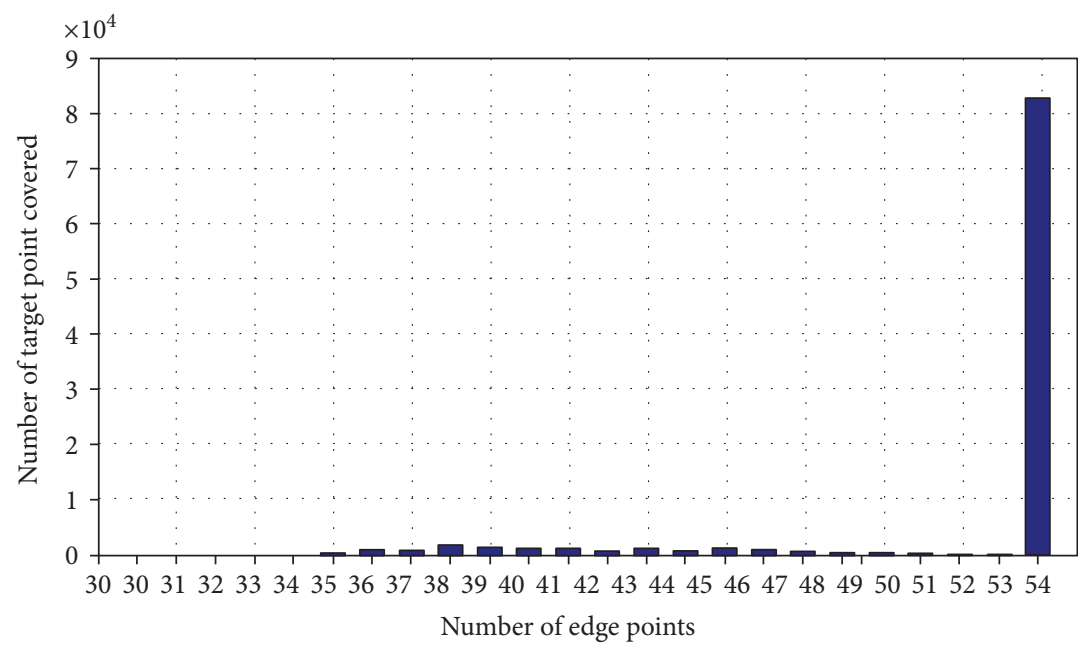

FIGURE 11: Coverage results of a circular area when the sensor angle is $7.7^{\circ}$. 


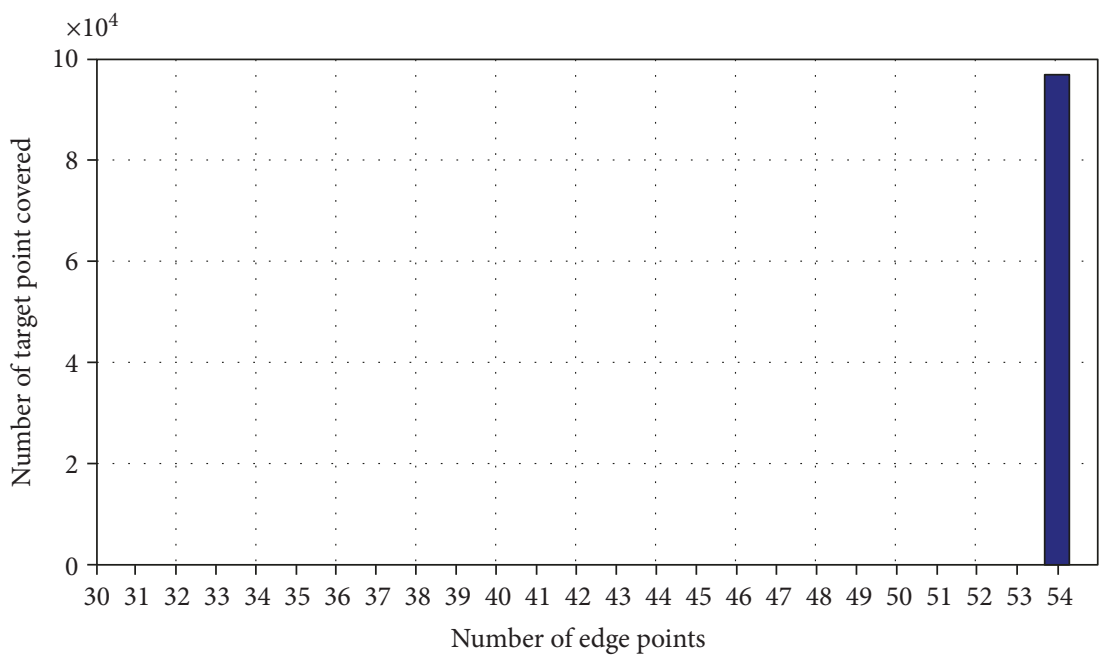

Figure 12: Coverage results of a circular area when the sensor angle is $8^{\circ}$.

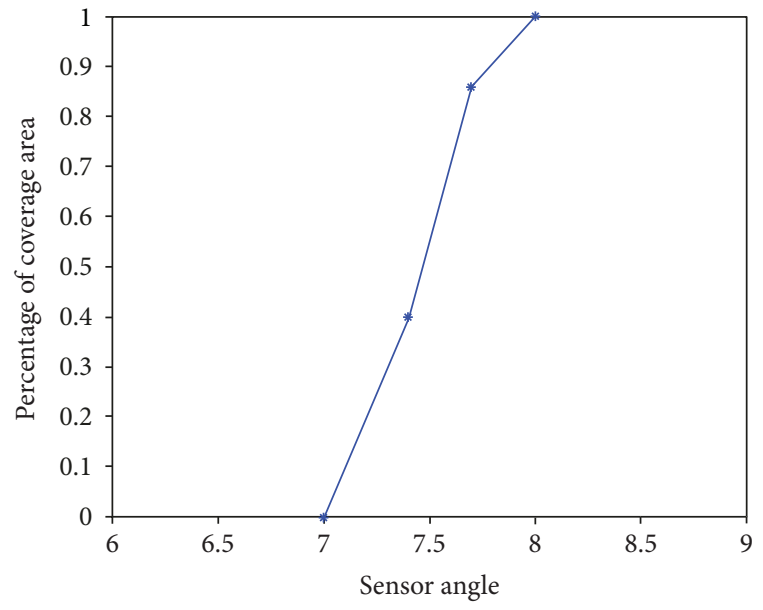

FIgURE 13: Percentage change of area coverage.

should be calculated with respect to the requirement of the complete coverage from satellite 2 . From case results shown in Table 14, we know that the minimum coverage radius of satellite 2 for coverage requirement is $1684.017928 \mathrm{~km}$.

\section{Results and Discussion}

Above cases have shown the influence of orbital element errors on coverage calculation. It is obvious that the minimum angle requirement is larger when considering error of orbital elements. From case 1, it can be seen that the influence of orbital element error on coverage is more significant when the required geocentric angle is smaller. As the required geocentric angle increases, the impact of orbital element error on coverage will be smaller and smaller. From case 2, an interesting phenomenon can be seen that when true anomaly is about $180^{\circ}$, and it is a turning point for the changes of axial length and axial ratio. Cases 3-5 also show the analysis results of different coverage problems based on the Monte Carlo simulation for area targets. These cases show that
TABLE 14: Minimum coverage radius for each target.

\begin{tabular}{lcc}
\hline Satellite & Point target & Minimum covering radius $(\mathrm{km})$ \\
\hline 1 & A & 669.590510 \\
2 & A & 1684.017928 \\
2 & B & 660.448878 \\
3 & B & 1659.206737 \\
\hline
\end{tabular}

under the case settings in this paper, the extra requirements of sensor angle grows by about $14.3 \%$. The analysis implemented in this paper is based on the fixed positions of satellite or constellation. Hence, in future research, this work can be extended to analyze the real-time coverage error related to positioning uncertainty.

\section{Conclusions}

In this paper, we present a method of accurately calculating the constellation coverage when considering position uncertainty. The position uncertainty of satellite is shown by an "error ellipsoid," whose spatial shape is also analyzed in the paper. For the illustration of proposed method, coverage calculations for a single satellite and constellation are both considered to carry out the coverage cases. The cases show that when considering the position uncertainty related to influence of orbital error, in order to surely cover the target points, the covering radius needs to be larger than not considering position uncertainty. The work presented in this paper also has important reference value for the reliability analysis of constellation design, which can effectively deal with the influence of position uncertainty.

\section{Conflicts of Interest}

The authors declare that there is no conflict of interests regarding the publication of this paper. 


\section{Acknowledgments}

This work is supported by National Natural Science Foundation of China under Grant no. 61472375, the 13th Five-Year Pre-Research Project of Civil Aerospace in China, the Joint Funds of Equipment Pre-Research and Ministry of Education of the People's Republic of China under Grant no. 6141A02022320, Fundamental Research Funds for the Central Universities under Grant nos. CUG2017G01 and CUG160207, and the Reform and Research of Graduate Education and Teaching under Grant no. YJS2018308.

\section{References}

[1] J. Cote, Maximizing Satellite Coverage, Pacific Yachting, 2012.

[2] K. W. Yung and D. C. Chang, U.S. Patent No. 7,198,230, U.S. Patent and Trademark Office, Washington, DC, USA, 2007.

[3] J. E. Draim, "A common-period four-satellite continuous global coverage constellation," Journal of Guidance, Control, and Dynamics, vol. 10, no. 5, pp. 492-499, 1987.

[4] Z. Lian, Y. Tan, Y. Xu, and J. Li, "Static and dynamic models of observation toward Earth by agile satellite coverage," in Proceedings of International Workshop on Planning and Scheduling for Space, pp. 1-6, Darmstadt, Germany: ESOC, 2011.

[5] H. B. Wang, F. Xue, and C. J. Liu, "Test method of satellite coverage area and its engineering application," Radio Communications Technology, vol. 3, p. 19, 2010.

[6] J. A. Ojeda Romero, Dual Satellite Coverage using Particle Swarm Optimization Doctoral dissertation, Virginia Tech, 2014.

[7] X. Huang and G. Dai, "An optimal design of constellation of multiple regional coverage based on NSGA-II," International Journal of Advancements in Computing Technology, vol. 4, no. 21, pp. 295-303, 2012.

[8] E. S. Fekpe, T. K. Windholz, and K. Beard, "Probabilistic approach for modeling and presenting error in spatial data," Journal of Surveying Engineering, vol. 135, no. 3, pp. 101$112,2009$.

[9] E. D. Kaplan, "Understanding GPS: principles and application," Journal of Atmospheric and Solar-Terrestrial Physics, vol. 59, no. 5, pp. 598-599, 2005.

[10] P. Misra and P. Enge, "Global Positioning System: Signals, Measurements and Performance," Ganga-Jamuna Press, 2011.

[11] W. Nelson, Use of Circular Error Probability in Target Detection, MITRE Corporation, Bedford MA, USA, 1988.

[12] J. C. Roh and D. W. Waters, "Position and velocity uncertainty metrics in GNSS receivers," US Patent US8525727, 2013.

[13] E. M. Mikhail and F. E. Ackermann, Observations and Least Squares, Harper and Row, New York, NY, USA, 1976.

[14] M. Nomura and T. Tanaka, "GPS positioning method under condition of only three acquired satellites," SICE Journal of Control, Measurement, and System Integration, vol. 2, no. 4, pp. 206-212, 2009.

[15] F. D. Nunes, F. M. G. Sousa, and J. M. N. Leitao, "Performance analysis of altimeter-aided GNSS receiver for indoor scenarios," in The 7th Conference on Telecommunications, Santa Maria da Feria, Portugal, May 2009.

[16] M. Wang, L. Ma, L. Zhang, and H. Ji, “Analysis of horizontal positioning error distribution of the Chinese area positioning system," Astronomical Research \& Technology, vol. 9, no. 3, pp. 522-526, 2012.
[17] S. Alfano, "Relating position uncertainty to maximum conjunction probability," Journal of the Astronautical Sciences, vol. 53, no. 2, pp. 193-205, 2004.

[18] Z. Qingzhu, Z. Jun, Z. Yanbo, and L. Wei, "Navigation accuracy category-position models and estimate position uncertainty calculations for tis-b system," Chinese Journal of Aeronautics, vol. 22, no. 4, pp. 419-425, 2009.

[19] Y. L. Shi, Q. M. Cui, S. Q. Cao, and X. F. Tao, "Distributed location model and algorithm with position uncertainty," The Journal of China Universities of Posts and Telecommunications, vol. 21, no. 2, pp. 48-56, 2014.

[20] D. A. Trifonov, "Position uncertainty measures on the sphere," in Proceedings of the Fifth International Conference on Geometry, Integrability and Quantization, pp. 211-224, Institute of Biophysics and Biomedical Engineering, Bulgarian Academy of Sciences, 2004. 


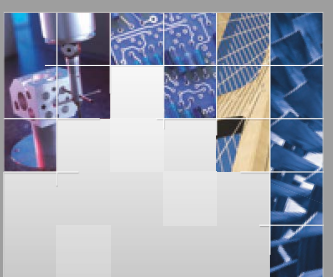

\section{Enfincering}
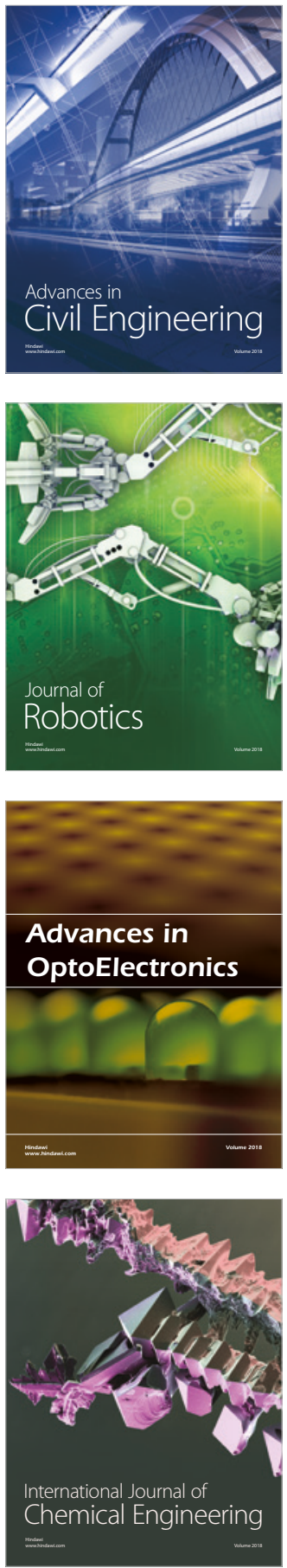

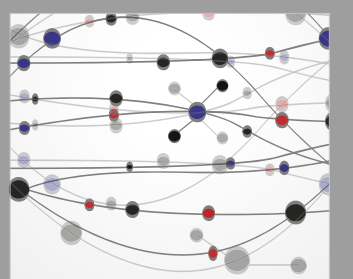

\section{Rotating \\ Machinery}

The Scientific World Journal

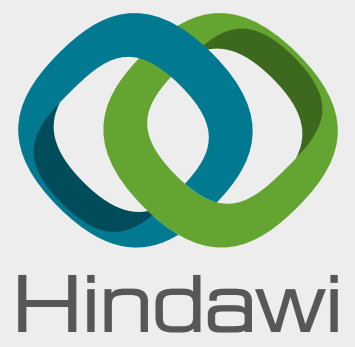

Submit your manuscripts at

www.hindawi.com
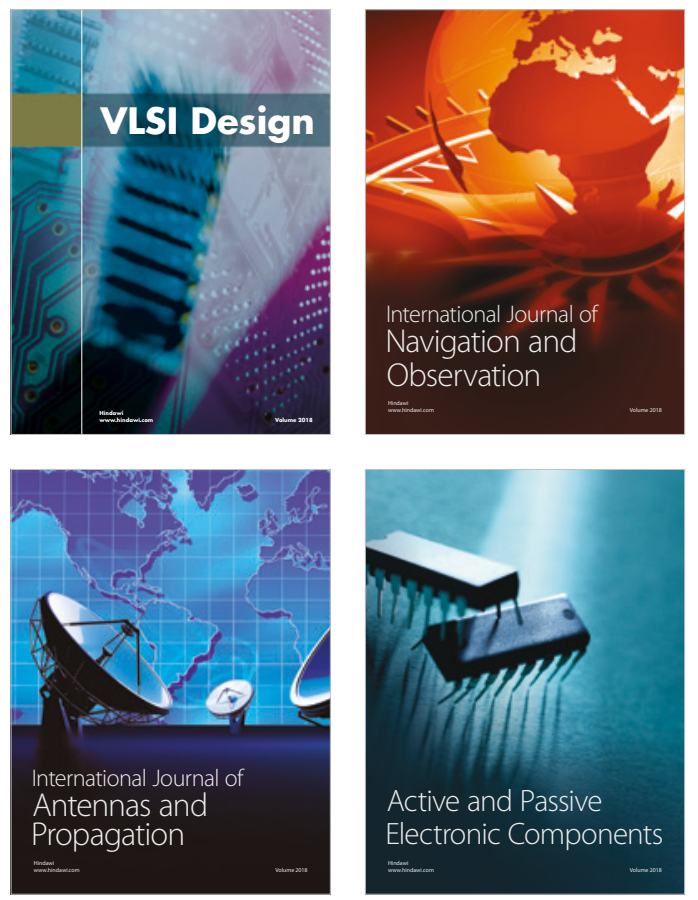
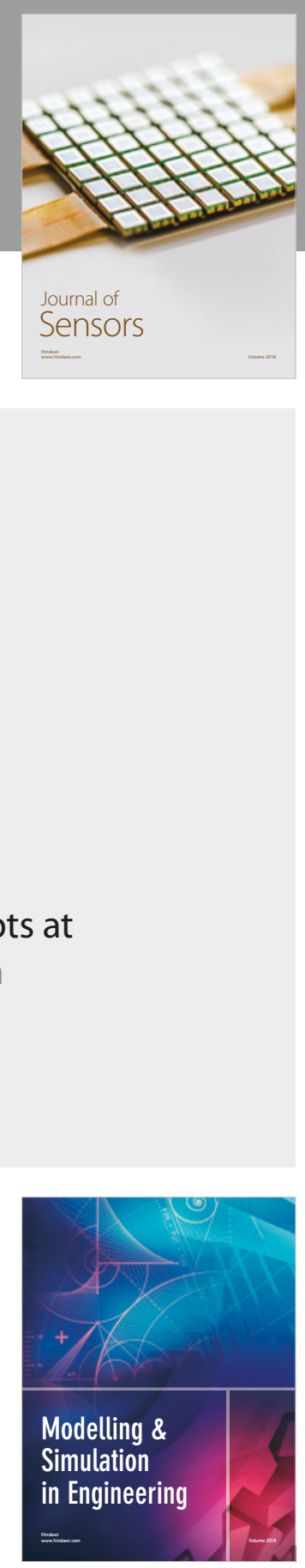

\section{Advances \\ Multimedia}
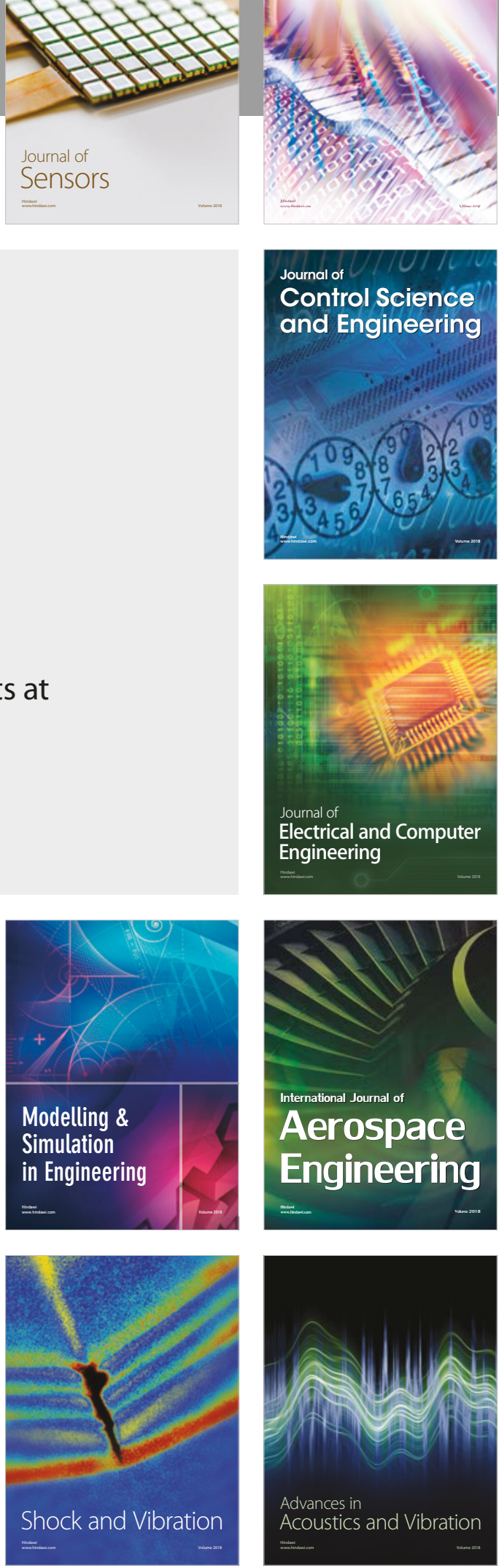